\title{
PROTOTIPAGEM 3D APLICADA A CIRURGIAS DE IMPLANTES DENTÁRIOS
}

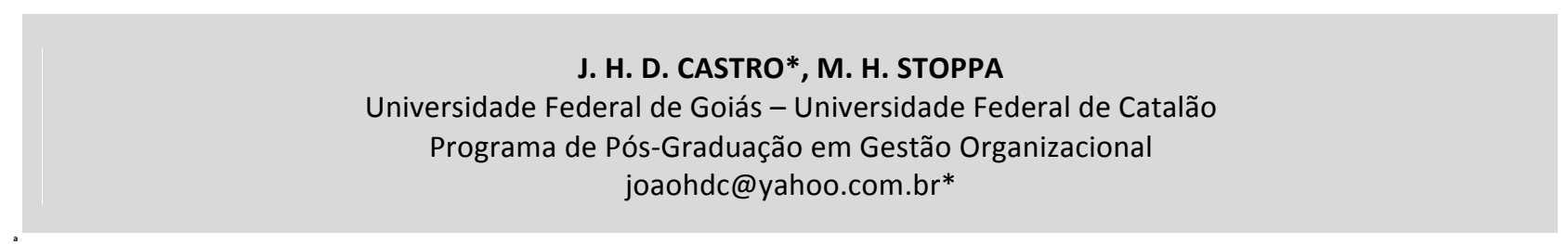

Submetido 03/03/2018 - Aceito 22/12/2018

DOI: $10.15628 /$ holos.2018.7039

\section{RESUMO}

A perda dental é um dos grandes problemas da Odontologia. Com os avanços tecnológicos, o implante dentário veio para realizar reposições de dentes extraídos. O uso de impressões 3D trouxe a possibilidade da reprodução das arcadas dentárias de forma fidedigna, graças ao programa InVesalius que processa imagens tomográficas transformando-as em um arquivo capaz de reproduzir por meio de impressão um protótipo dimensionalmente fiel. Com relação ao Odontólogo, a prototipagem ofereceu grandes benefícios, tais como: clareza no planejamento cirúrgico e segurança na execução dos procedimentos, além de permitir ao paciente elucidação prévia do processo, diminuindo inúmeras insatisfações e dúvidas quanto ao tratamento proposto. Aliados às vantagens oferecidas ao dentista e ao paciente, pelo protótipo 3D, o fator custo é de suma importância devido à sua irrelevância quanto ao valor total de um implante dentário frente aos benefícios oferecidos decorrentes do uso da prototipagem 3D. Portanto, este trabalho apresenta o processo de geração de um modelo tridimensional por meio do software Invesalius e a sua impressão, com posterior teste de implante dentário.

PALAVRAS-CHAVE: exodontia, InVesalius, prototipagem 3D, implante dentário, cirurgia.

\section{D PROTOTYPING APPLIED TO DENTAL IMPLANT SURGERIES}

\section{ABSTRACT}

Dental loss is one of the major problems of dentistry. With the technological advances, the dental implant came to make repositions of extracted teeth. The use of $3 D$ prints has provided a reliable reproduction of dental arches thanks to the InVesalius program that processes tomographic images, transforming it into a file capable of reproducing a dimensionally faithful prototype. With regard to the dentist, prototyping offers great benefits, such as: clarity in surgical planning and safety in the execution of procedures, besides allowing the patient, the approval process, as well as disapproval of dissatisfactions and doubts about the proposed treatment. In addition to the advantages offered to the dentist and the patient by the 3D prototype, the cost factor is extremely important due to its irrelevance when compared to the total value of a dental implant compared to the
\end{abstract}

benefits offered by using 3D prototyping. Then this work presents the process of generation of a 3dimensional model through InVesalius software and its printing, with posterior dental implant test.

KEYWORDS: extraction, InVesalius, 3D prototyping, dental implant, surgerie. 


\section{INTRODUÇÃO}

Devido aos avanços tecnológicos, na década de 80 , a prototipagem rápida foi relevantemente estudada, e atualmente é chamada de manufatura aditiva. Diversos autores se interessaram por projetos nessa área, orbitando acerca das necessidades de redução de prazos de manufatura, exigidas pelos consumidores. Esse foi enfoque de Cunico (2015), no tocante ao incremento de materiais inovadores. Nesse contexto, o processo da prototipagem rápida foi desenvolvido com o intuito de juntar partes para formar um objeto real. Pallarolas (2013) apresentou uma variada análise e tomada de decisões quanto a projetos interdisciplinares, cujo objetivo era encontrar o melhor modelo, o qual, se realizado de forma adequada, agregaria valor ao produto.

$\mathrm{Na}$ Odontologia, este processo teve um gradual crescimento de aplicação nas últimas três décadas, com a finalidade de reproduzir de forma fiel uma arcada dentária; principalmente, motivado pelo fato dos implantes dentários necessitarem de um meticuloso planejamento para se atingir uma excelência no que se refere à osseointegração. Daí, a necessidade de um modelo tridimensional construído por impressão 3D, de modo a facilitar a gestão do trabalho do implantodontista, desde o planejamento, passando pela cirurgia até a reabilitação final.

Essa pesquisa vem apresentar um processo que permite obter melhores resultados, evitando a ocorrência de repetições, riscos e falhas. Para atingir esse objetivo, foi necessário pesquisar e avaliar a viabilidade de construção de um protótipo que reproduziria de modo fiel arcadas dentárias, utilizando impressoras 3D do Laboratório de Modelagem e Prototipagem 3D (LaMoP3D) da UFG Regional Catalão, tomando por base imagens tomográficas disponíveis no repositório público de imagens tomográficas www.dicomlibrary.com.

Foi imprescindível a avaliação de imagens tomográficas e tipos de tomógrafos para posterior utilização dessas imagens no software InVesalius que, a partir de arquivos DICOM (um acrônimo para Digital Imaging and Communications in Medicine, que costuma ser o padrão mais comum para recebimento de digitalizações médicas, especialmente, imagens oriundas de tomógrafos ou aparelhos de ressonância magnética), produzidos pelos tomógrafos, é seja capaz de gerar imagens 3D, geometricamente, idênticas aos modelos reais. Na sequência, efetuadas adequações e edições dessas imagens, foi construído o protótipo por impressão tridimensional, em especial pelo processo de deposição de material, o que permitiu a realização de um implante prévio no protótipo impresso, seguindo todos os passos e normas técnicas do procedimento real.

\section{CONTEXTUALIZAÇÃO}

\subsection{História da Implantodontia}

A Odontologia preocupa-se com as usuais extrações motivadas, principalmente, pela cárie dental, aliada a outros fatores, tais como traumatismo dentário e doenças periodontais, paralelamente, ao alto impacto financeiro existente no caso de implantes dentários (Barbato, 
Nagano, Zanchet, Boing, \& Peres, 2007). Ademais, existem os agravantes decorrentes da dificuldade do tratamento pelo sistema de saúde pública.

Segundo McClarence (2003), os primeiros experimentos relativos a implantes dentários, foram efetuados pelo professor Brånemark na década de 50. Porém, efetivamente, o início dos implantes dentários datam de 1965 , quando foi possível realizar o primeiro implante em humanos na Universidade de Gotemburgo.

\subsection{Tomografia e o InVesalius}

O vocábulo tomografia, segundo Silva (2004), procede da união de duas palavras gregas, a saber tomos, que significa "cortes" ou "fatias" e grafos, a qual significa "escrever" ou "escrita".

Na década de 70, a primeira imagem clínica de uma tomografia computadorizada foi feita pelo engenheiro eletroquímico Godfrey Newbold Hounsfield (ARELLANO, 2001).

Mais recentemente, González-Martín e Veltri (2017) analisaram, tridimensionalmente, um implante com osso vestibular, utilizando a tomografia computadorizada de feixe cônico. Após determinado período, a reconstrução virtual tridimensional pôde ser avaliada com relação ao nível ósseo marginal vestibular e volume do rebordo vestibular porque os dados da tomografia concederam esses dados anatômicos.

O programa InVesalius permite criar um modelo tridimensional a partir de imagens obtidas por tomografia e ressonância magnética, utilizando os arquivos do tipo DICOM: esse software possibilita gerar modelos 3D de qualquer parte do corpo humano.

O software, que se encontra no portal do software público, foi desenvolvido pelo Centro de Tecnologia da Informação Renato Archer, equivalente a um centro de pesquisa do Ministério da Ciência e Tecnologia do Brasil. O InVesalius é disponibilizado em diversas línguas e de uso integralmente livre, e, por isso, é de grande auxílio à prototipagem 3D, em seres humanos.

\subsection{Impressões 3D}

Existem diversos tipos de impressão 3D: a Estereolotografia que utiliza um líquido foto polímero sensível à luz ultravioleta que solidifica a região a ela exposta; a Sinterização Seletiva a Laser refere-se a uma impressão baseada em laser aplicado a materiais em pó, fundindo as partículas entre si, formando uma fina camada sólida; a Modelagem por Fusão e Deposição de material (Fused Deposition Modeling - FDM), a qual utiliza filamentos plásticos que são derretidos e depositados em camadas, formando o objeto.

Monteiro (2015) afirma que esse modelo é o mais vantajoso pelo baixo custo e facilidade de funcionamento, sendo que a maior vantagem apresentada se refere à quantidade de materiais a ser impressos.

Na verdade, esses processos de impressão descritos aqui têm um princípio comum, que é a construção camada a camada, sendo este último o mais comum e utilizado, essencialmente, devido ao seu baixo custo de equipamentos e insumos. Esse processo de impressão é comumente conhecido por manufatura aditiva. 
Ferreira Neto (2017) exemplifica bem este processo, que utiliza um software CAD (computer-aided design - desenho assistido por computador), o qual é imprescindível para produzir e obter um protótipo 3D. Na sequência, o objeto virtual gerado é processado por um software CAM (computer-aided manufacturing - manufatura assistida por computador) que fornece as instruções para impressora 3D, gerando um arquivo digital com as coordenadas dos eixos $(x, y, z)$ da peça. Finalmente, de posse dessas instruções, será impresso o protótipo idealizado (Figuras 1 e 2 ).

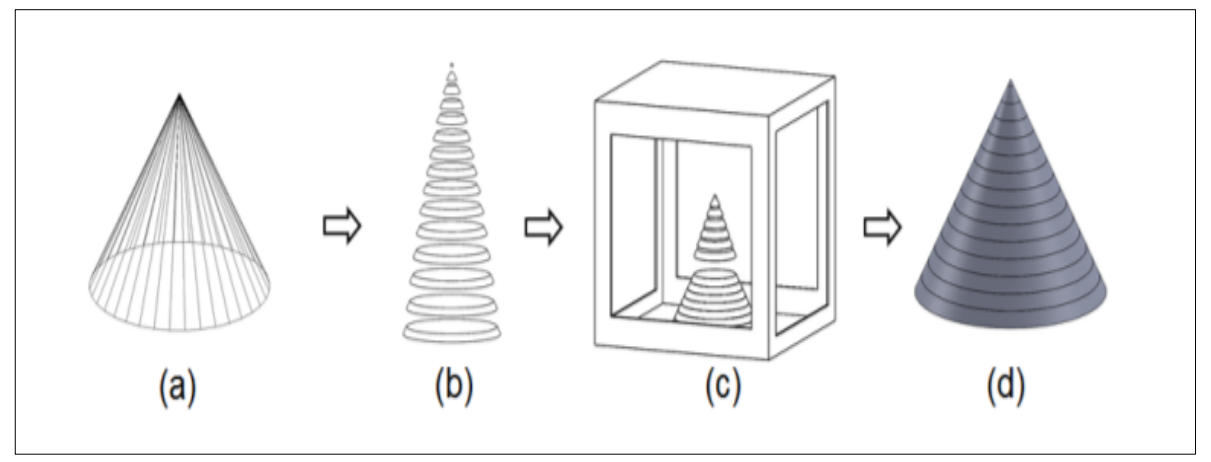

Figura 1: Etapas para impressão 3D - (a) Modelo Virtual; (b) Modelo Fatiado; (c) Fabricação; (d) Objeto. Fonte: Ferreira Neto (2017).

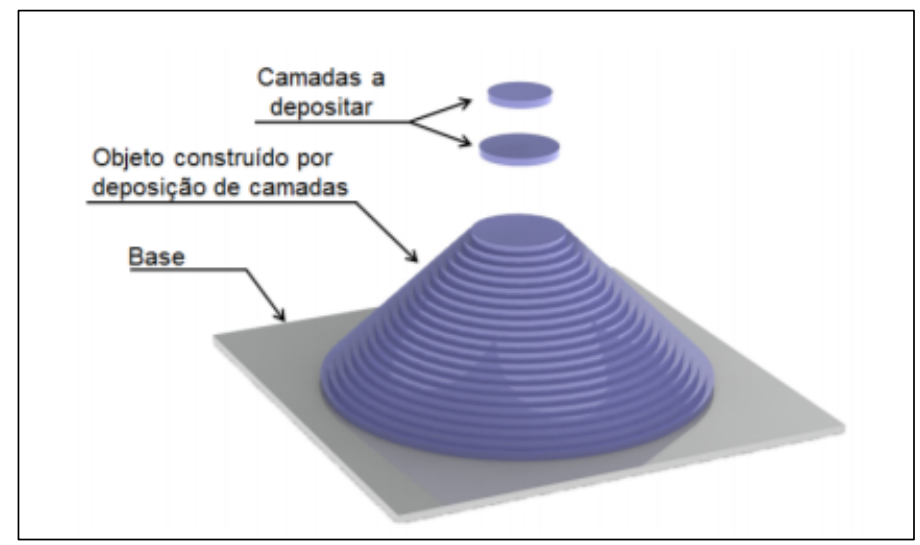

Figura 2: Construção por camadas. Fonte: Ferreira Neto (2017).

\subsection{Projeto RepRap}

O desenvolvimento e popularização da tecnologia FDM, teve a grande contribuição de Adrian Bowyer na Universidade de Bath no Reino Unido, que construiu um projeto para produção de impressoras denominado RepRap, que pode fabricar inúmeras peças através de uma impressora 3D funcional, inicialmente a baixo custo.

Conforme relato de Pallaropas (2013), em consonância com Ferreira Neto (2017), esse projeto conseguiu vários clientes e, rapidamente, vários modelos foram desenvolvidos para se obter baixo custo, em que a característica de projeto livre fez qualquer pessoa ter acesso para contribuir com as melhorias da FDM, permitindo maior acessibilidade de usuários. A Figura 3 exibe modelos de impressoras FDM do Laboratório de Modelagem e Prototipagem 3D (LaMoP3D), da Universidade Federal de Goiás, Regional Catalão, onde foi desenvolvida esta pesquisa. 


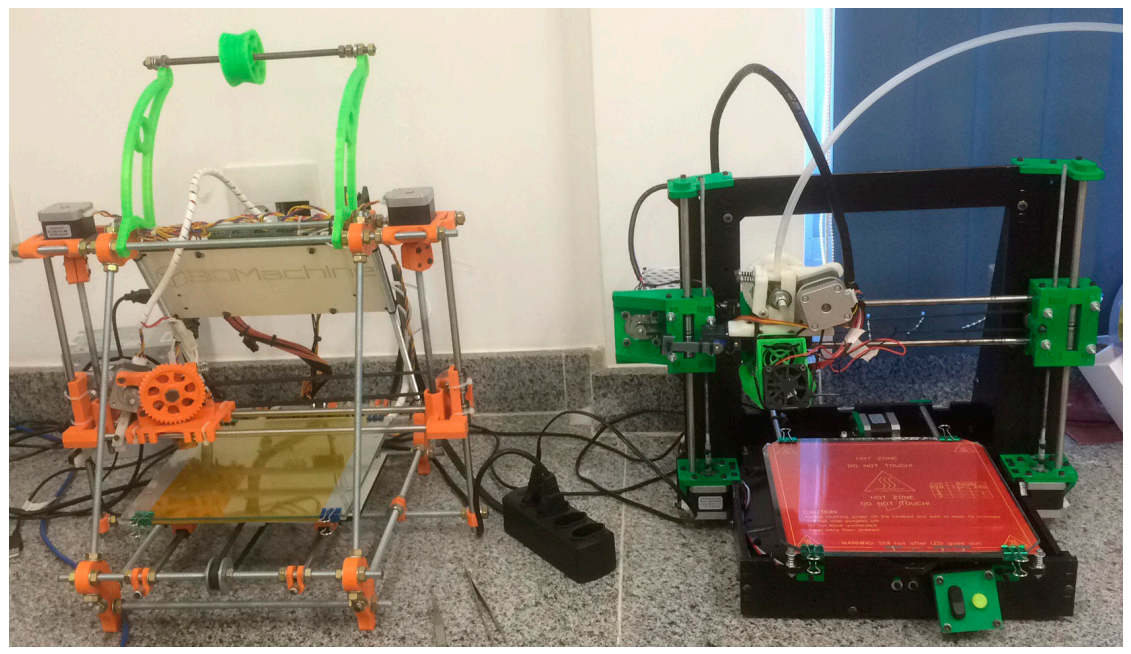

Figura 3: Impressoras FDM. Fonte: autoria própria.

\subsection{Prototipagem Aplicada às Áreas Médicas e Odontológicas}

A finalidade essencial da prototipagem, aplicada à Medicina e Odontologia, é diminuir as complicações decorrentes do diagnóstico, bem como auxiliar o planejamento pré-cirúrgico de modo a obter um excelente tratamento na ocasião de se executar procedimentos cirúrgicos e protéticos.

Ventola (2014) afirma que a prototipagem 3D começou a ser utilizada nessas ciências no início desse século, primeiramente, em implantes dentários.

A partir de imagens de tomografia computadorizada reproduzidas em impressão 3D, essas apresentam vantagens relacionadas ao custo, produtividade e confeç̧ão de modelos de ossos e gengivas coloridos idênticos, tendo algumas desvantagens como reprodução de estruturas finas (Meurer et al., 2008).

De igual modo, Margonar et al. (2012) elaboraram um protótipo 3D, usando a tomografia computadorizada, o que permitiu um planejamento mais adequado, melhores procedimentos e redução de tempo cirúrgico.

Assim, é evidente o crescimento da utilização da prototipagem 3D como ferramenta auxiliar utilizado com o intuito de melhorar o procedimento cirúrgico, além de proporcionar maior conforto e menores complicações pós-cirúrgicas aos pacientes.

\section{METODOLOGIA}

O presente estudo apresenta o desenvolvimento da reprodução da arcada dentária de pacientes desdentados total ou parcialmente, através da impressão de um protótipo, permitindo uma representação física tridimensional dessa arcada. Foram utilizados arquivos tomográficos de arcadas disponíveis no site www.dicomlibrary.com, que é um banco de dados público de arquivos DICOM, gerados em tomógrafos e aparelhos de ressonância magnética, para fins de pesquisa, ciência e educação. Este repositório é um meio de compartilhar arquivos e imagens da Medicina e Odontologia com finalidade educativa e científica, inteiramente gratuita. 
Em posse das imagens adquiridas dos aparelhos tomográficos, o InVesalius (Figura 4), é capaz de fazer a leitura dos arquivos DICOM e gerar o modelo CAD tridimensional da arcada dentária (Figura 5).
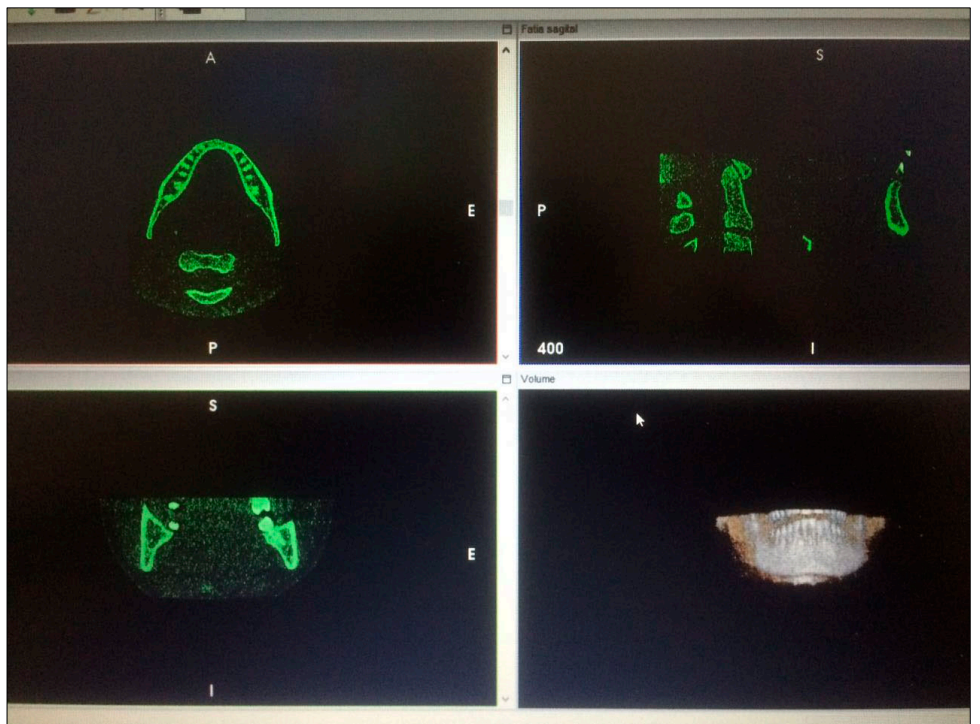

Figura 4: Programa InVesalius. Fonte: autoria própria.

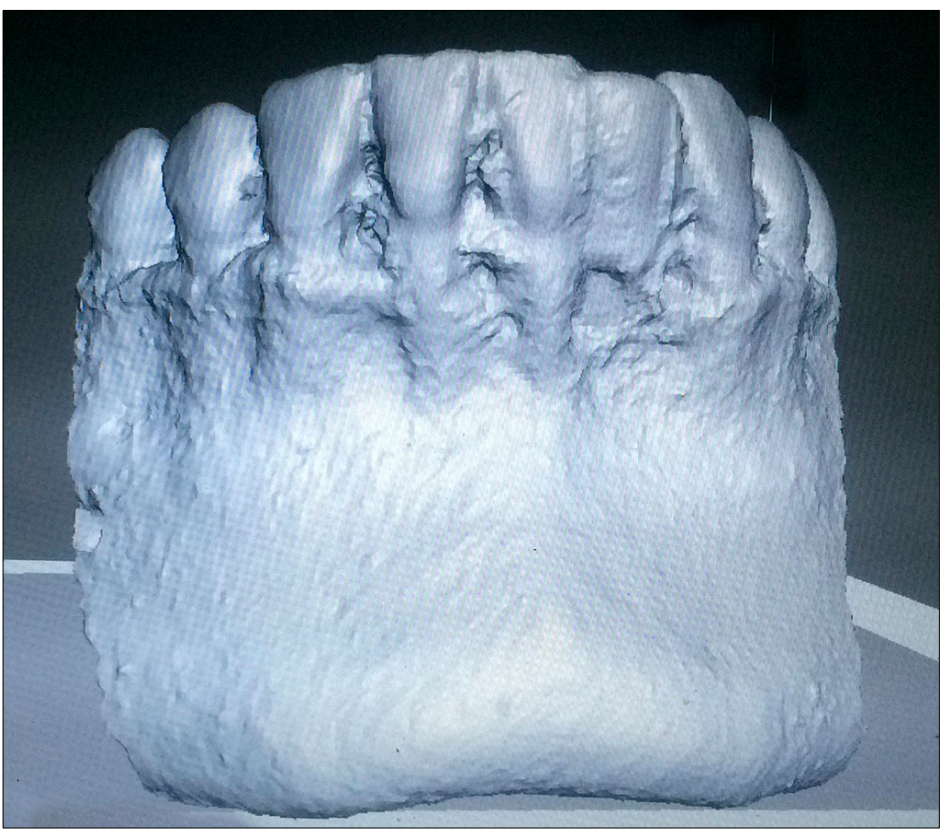

Figura 5: Modelo 3D. Fonte: autoria própria.

Na sequência, o modelo 3D produzido nas impressoras 3D do LaMoP3D da UFG Regional Catalão (Figura 6). 


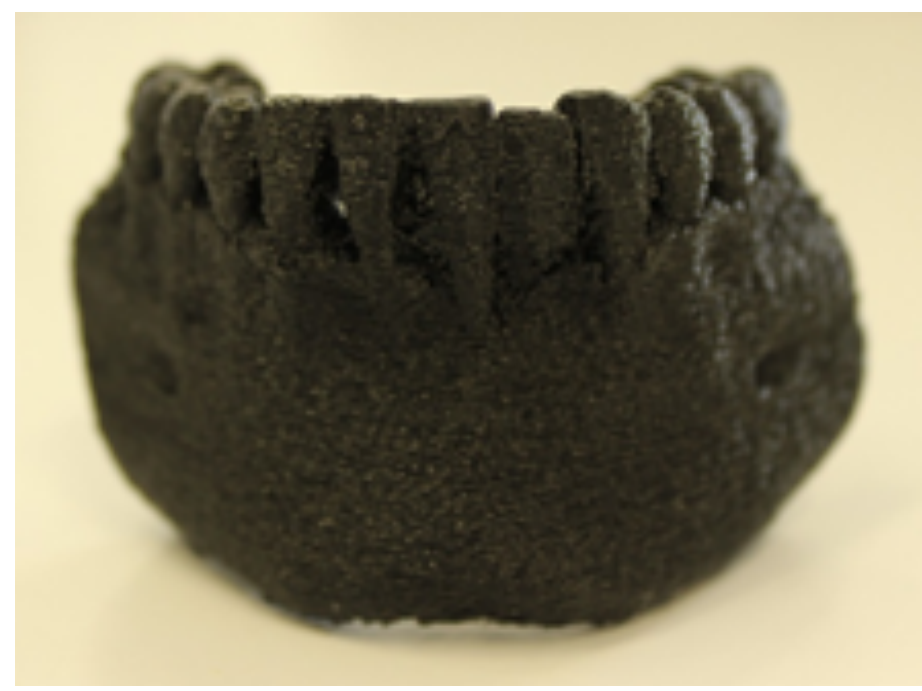

Figura 6: Impressão da arcada dentária, produzida no LaMoP3D. Fonte: autoria própria.

O protótipo 3D permite a elaboração de um planejamento mais eficaz para quaisquer outros procedimentos inclusos nas cirurgias odontológicas reduzindo dificuldades e erros que possam ser originados no processo.

Inicialmente, fez-se a instalação de todos os aparelhos necessários para a execução de um implante experimental no LaMoP3D, tais como, o kit cirúrgico, o motor e contra ângulo.

O polímero utilizado na impressão do protótipo foi o PLA (Ácido Polilático) que é um ótimo material dentre os tipos de filamentos disponíveis por ser biodegradável e de origem vegetal, além de não necessitar de altas temperaturas para a extrusão; esse se aproximou fisicamente do osso maxilar, considerando, por exemplo, a densidade e proporciona uma oportunidade para planejamento prévio da cirurgia.

Os procedimentos de preparação pré-cirúrgica foram conduzidos para a execução do implante dentário no elemento 31 do protótipo (Figura 7a). Uma vez executado o implante, observou-se que a simulação da cirurgia é equivalente à real, permitindo uma visão adequada e avaliação prévia sem que o paciente seja exposto a experimentação real em consultório odontológico (Figura 7b).

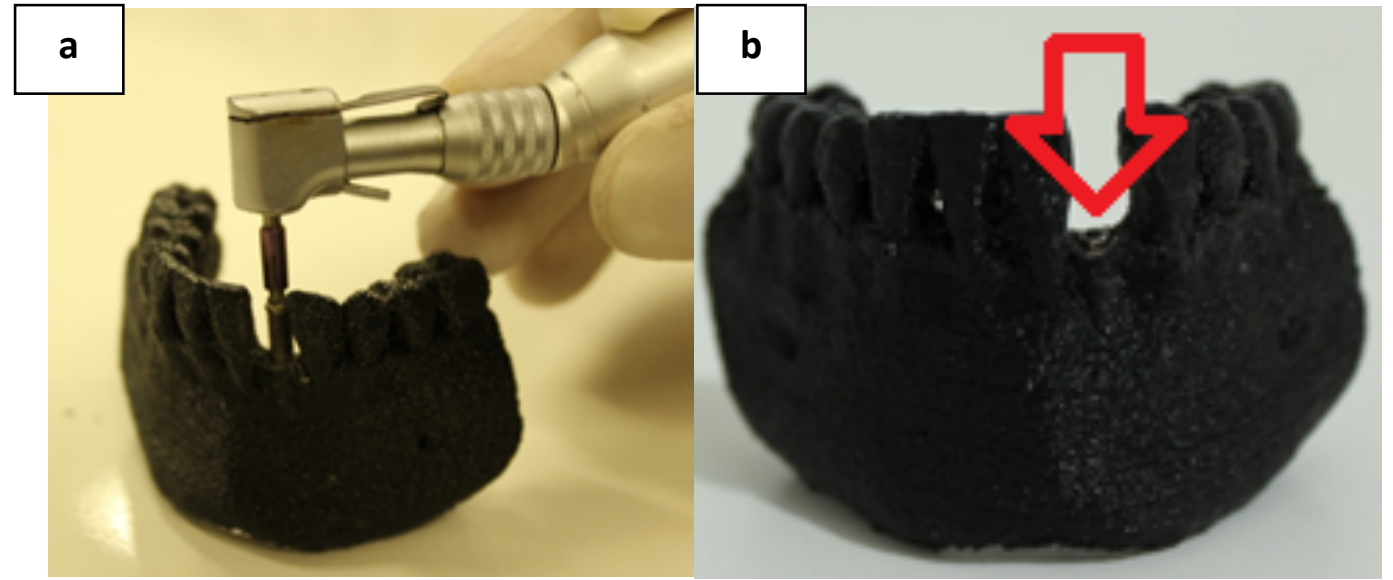

Figura 7: (a) execução do implante dentário; (b) implante instalado no protótipo. Fonte: autoria própria. 
Esses procedimentos permitem mitigar falhas por meio de planejamento anterior à sua execução em consultório, viabilizando um excelente resultado final, principalmente, por permitir ao paciente avaliar, visualmente, como ficará o dente que ainda será implantado (Figura 8).

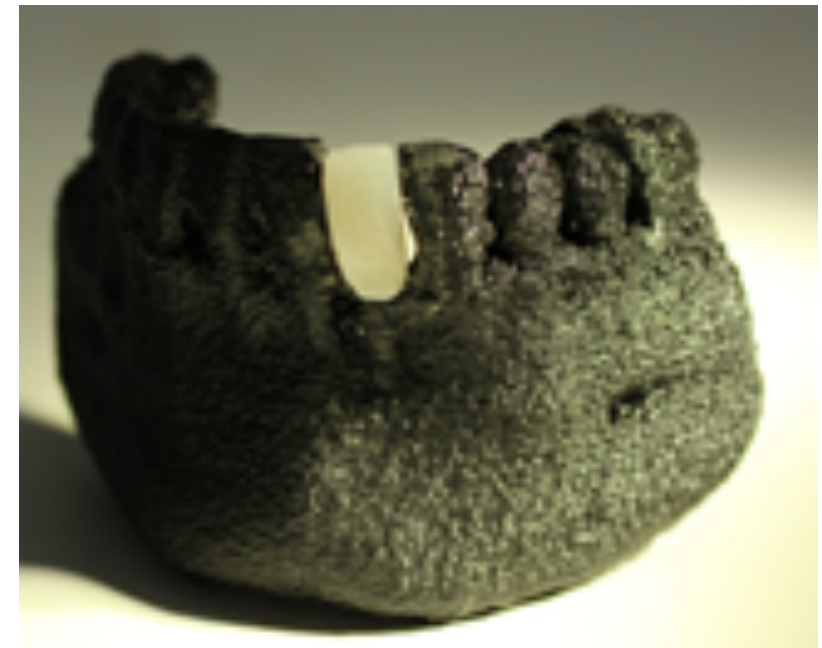

Figura 8: Imagem do dente confeccionado. Fonte: autoria própria.

Por meio dos resultados apresentados nesse trabalho, vale ressaltar as vantagens decorrentes, tanto para o odontólogo quanto ao suposto paciente.

Em relação ao primeiro, determinados fatores são relevantes, tais como, diminuição da hora clínica, facilidade quanto à visualização prévia cirúrgica, decréscimo de fatores prejudiciais, sejam por falhas ou situações imprevistas, ou mesmo, riscos por ter o protótipo previamente confeccionado e possibilidade de opções quanto à escolha do implante.

No que se refere ao paciente, a impressão 3D permite que este possa avaliar fisicamente o tratamento proposto pelo cirurgião. Além disso, em se tratando do custo, o valor do protótipo é insignificante quando comparado com o valor total do implante. No tangente às condições, traumáticas ou psicológicas, ocasionadas pelos usuais tratamentos dentários, o protótipo oferece mais segurança por sua visualização real e pode ser uma ferramenta didática auxiliar para o cirurgião explicar os detalhes do procedimento. E, finalmente, para ambos é possível interagir quanto aos procedimentos de forma mais elucidativa graças à prototipagem.

\section{CONCLUSÕES}

Com o desenvolvimento tecnológico, a confecção do protótipo 3D auxilia no planejamento das cirurgias odontológicas, especialmente, no que se refere aos implantes dentários, o que facilita a ação do cirurgião com uma prototipagem análoga às arcadas dentárias, sendo possível prever a reabilitação com implante dental de maneira mais precisa.

O presente artigo visa também mostrar a importância da prototipagem obtida por meio do software InVesalius o qual é livre para pesquisas e, ainda, analisa e reproduz as imagens tridimensionais para posterior uso em impressora 3D. 
Com essa abordagem, espera-se que esse trabalho contribua para melhoria nas cirurgias odontológicas, especificamente, nos implantes dentários, de igual modo, ao odontólogo porque Ihe permitirá maior clareza na determinação dos seus planejamentos cirúrgicos, bem como segurança para execução destes.

Em contrapartida, permitirá ao paciente elucidação, minimizando-lhe quaisquer futuras insatisfações e sanando dúvidas quanto ao tratamento proposto. É valido, também, enfatizar que o protótipo 3D, quanto ao fator financeiro, será inferior a $3 \%$ do valor de um implante, sendo irrelevante o custo benefício tendo em vista as vantagens aqui apontadas porque evitará inúmeras repetições e falhas inerentes a tal procedimento.

\section{REFERÊNCIAS}

Archer, R. (2017). InVesalius 3. Disponível a partir de https://www.cti.gov.br/pt-br/invesalius

Arellano, J. (2001). Tomografia computadorizada (tc) no diagnóstico e controle do tratamento das disfunções da articulação temporomandibular. J. Bras. Oclusão, ATM e Dor Orofacial, 1(4), 315-323.

Barbato, P. R., Nagano, H. C. M., Zanchet, F. N., Boing, A. F., \& Peres, M. A. (2007). Perdas dentárias e fatores sociais, demográficos e de serviços associados em adultos brasileiros: uma análise dos dados do estudo epidemiológico nacional (Projeto S Brasil 2002-2003). Cad. Saúde Pública, 23(8), 1803-14.

Cunico, M. W. M. (2015). Impressoras 3D: o novo meio produtivo. [S.I.]: Concep3d Pesquisas científicas.

Ferreira Neto, G. (2017). Modelagem e desenvolvimento de prótese de mão mecatrônica com acionamento individual de dedos (Dissertação de mestrado, Universidade Federal de Goiás, Catalão, Brasil).

González-Martín, O., \& Veltri, M. (2017). Cone beam analysis of the buccal bone associated witha dental implant: a tridimensional assessment case report. Quintessence International, 48(4), 339-344.

Margonar, R., Queiroz, T. P., Luvizuto, E. R., Santos, P. L., Wady, A. F., \& Paleari, A. G. (2012). Anterior tooth rehabilitation with frozen homogenous bone graft and immediately loaded titanium implant using computer-guided surgery. Journal of Craniofacial Surgery, LWW, 23(5), e470-e472. doi:10.1097/SCS.0b013e3182587516

McClarence, E. (2003). Close to the Edge: Brannemark and the Development of Osseointegration. [S.I.]: Quintessence London.

Meurer, M. I., Meurer, E., Lopes da Silva, J. V., Bárbara, A. S., Nobre, L. F., Oliveira, M. G. de, \& Silva, D. N. (2008). Aquisição e manipulação de imagens por tomografia computadorizadada região maxilofacial visando à obtenção de protótipos biomédicos. Radiologia Brasileira, 41(1), 49-54.

Monteiro, M. T. F. (2015). A impressão 3d no meio produtivo e o design: um estudo na fabricação de joias (Dissertação de mestrado, Universidade do Estado de Minas Gerais, Belo Horizonte, Brasil). 
Pallarolas, E. A. F. F. (2013). Revisão técnica de processos de manufatura aditiva e estudo de configurações geométricas, para estruturas de impressoras tridimensionais (Tese de doutorado, Universidade de São Paulo, São Paulo, Brasil).

Silva, A. C. (2004). Algoritmos para diagnóstico assistido de nódulos pulmonares solitários em imagens de tomografia computadorizada (Tese de doutorado, Pontifícia Universidade Católica do Rio de Janeiro, Rio de Janeiro, Brasil).

Ventola, C. L. (2014). Medical applications for 3d printing: current and projected uses. Pharmacy and Therapeutics, MediMedia, USA, 39(10), 704-711. 